\title{
The Effect of Slow Coronary Flow on Right and Left Ventricular Performance
}

\author{
Fatih Altunkas Fatih Koc Koksal Ceyhan Atac Celik Hasan Kadi \\ Metin Karayakali Kerem Ozbek Turgay Burucu Ahmet Ozturk Orhan Onalan \\ Department of Cardiology, Faculty of Medicine, Gaziosmanpasa University, Tokat, Turkey
}

\section{Key Words}

Right ventricular function - Left ventricular function .

Myocardial performance index · Slow coronary flow $\cdot$ Tissue

Doppler echocardiography $\cdot$ Coronary artery disease trol groups. Conclusion: This study showed that SCF affected LV functions echocardiographically and could cause partially reduced LV performance. In addition, SCF did not affect RV functions echocardiographically.

(c) 2013 S. Karger AG, Base

\begin{abstract}
Objective: To evaluate left and right ventricular functions using tissue Doppler echocardiography (TDE) and myocardial performance index (MPI) methods in patients with slow coronary flow (SCF) and to determine the relationship between these parameters and thrombolysis in myocardial infarction frame count in SCF patients. Subjects and Methods: Thirtyfive patients ( 20 males and 15 females) with SCF who underwent coronary angiography and 35 age- and sex-matched controls (14 males and 21 females) without SCF who underwent elective coronary angiography were enrolled in the study. Left ventricular (LV) and right ventricular (RV) functions were examined using conventional echocardiography and TDE. Results: LV systolic myocardial velocity (Sm), early myocardial velocity (Em), late myocardial velocity (Am), and Em/Am ratio were similar in both the SCF and control groups; however, isovolumetric relaxation time (IRT) was higher in the SCF group compared to the control group (IRT: $99 \pm 17$ vs. $88 \pm 20 ; p=0.01)$. In patients with SCF, LV MPI was higher than in the control group, but this was not statistically significant $(0.61 \pm 0.11$ vs. $0.56 \pm 0.12 ; p=0.07)$. The RV tricuspid annular velocities and MPI were similar in the SCF and con-
\end{abstract}

\section{Introduction}

Slow coronary flow (SCF) is a rare angiographic finding observed in patients with normal or near-normal coronary arteries and is characterized by slow contrast flow to the distal vascular structures during angiography [1]. Etiopathogenesis of SCF is still not clear. Previous studies indicate that microvascular reserve anomalies, increased vasoconstrictor response, decreased NO levels, inflammation, and adrenergic hyperactivation contribute to the etiopathogenesis of SCF [1-6]. There is very little information on the incidence of this disease, except in a study by Goel et al. [7] who report an incidence of $1 \%$.

The thrombolysis in myocardial infarction (TIMI) frame count (TFC) method is an objective method for evaluating coronary blood flow. This method measures the number of frames over which the contrast (dye) flows from the injection site to a predefined distal point [8]. Previous studies showed that in arteries with slow flow, TFC is significantly increased $[1,9,10]$.

\begin{tabular}{ll}
\hline KARGER & $\begin{array}{l}\text { ○ 2013 S. Karger AG, Basel } \\
1011-7571 / 14 / 0231-0034 \$ 39.50 / 0 \quad \text { Karger }\end{array}$ \\
E-Mail karger@karger.com & $\begin{array}{l}\text { This is an Open Access article licensed under the terms of the } \\
\text { Creative Commons Attribution-NonCommercial 3.0 Un- } \\
\text { ported license (CC BY-NC) (www.karger.com/OA-license), } \\
\text { applicable to the online version of the article only. Distribu- } \\
\text { tion permitted for non-commercial purposes only. }\end{array}$
\end{tabular}

Fatih Altunkas, MD

Gaziosmanpasa Universitesi

Arastirma Hastanesi Kardiyoloji AD

Eski rektorluk binas1, TR-60100 Tokat (Turkey)

E-Mail faltunkas@yahoo.com 
Fig. 1. Assessment of TFC value of the control group. a The first frame count of the LAD: the first frame used for TIMI frame counting is the first frame in which the dye fully enters the artery (arrow). b The last frame count of the LAD: the last frame is defined as the frame when the dye first enters the distal bifurcation of the LAD (arrow). TFC for this case was calculated as 20 .

Fig. 2. Assessment of TFC value in a patient with SCF. a The first frame count of the LAD: the first frame used for TIMI frame counting is the first frame in which the dye fully enters the artery (arrow). b The last frame count of the LAD: the last frame is defined as the frame when the dye first enters the distal bifurcation of the LAD (arrow). TFC for this case was calculated as 90 .
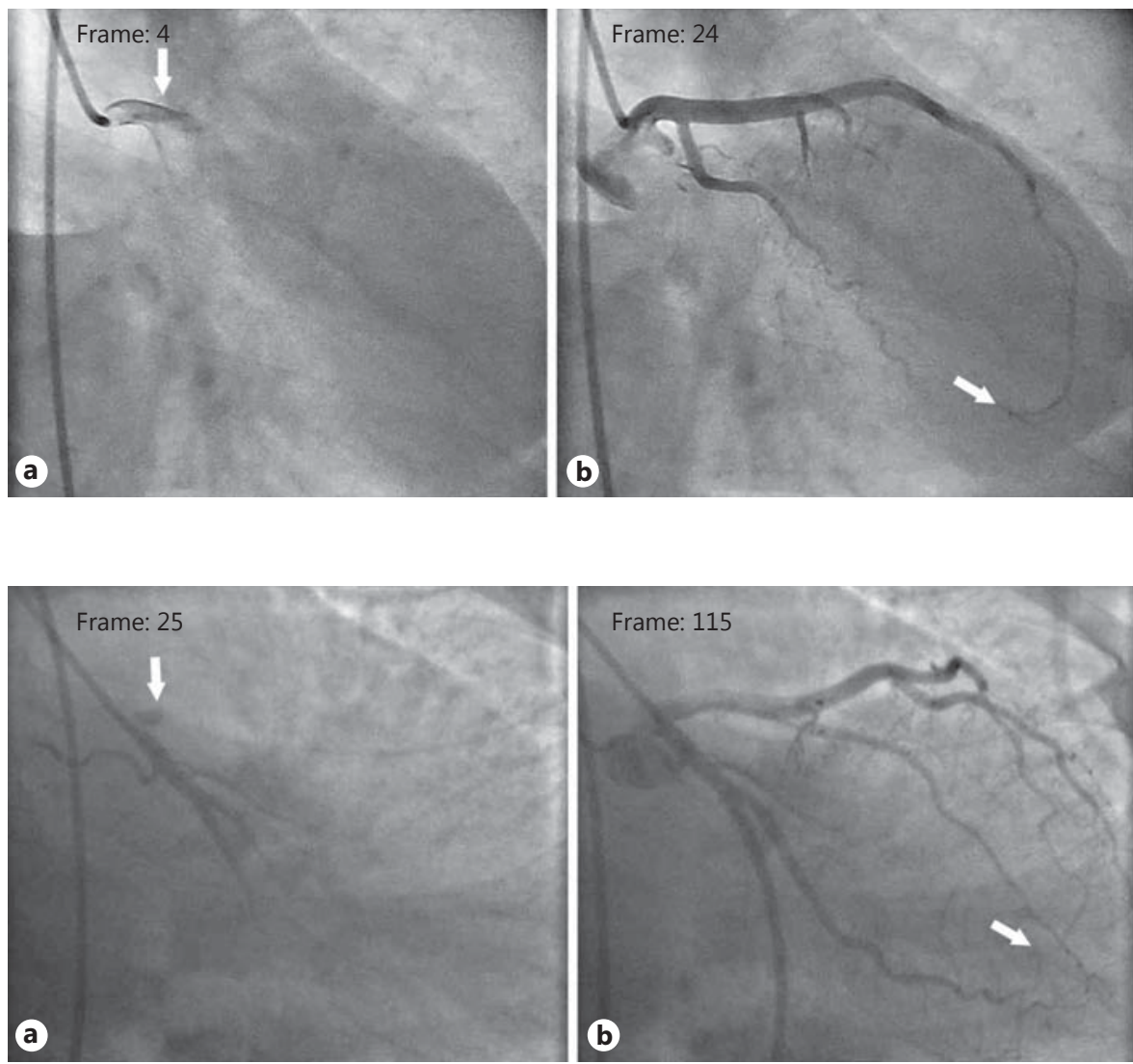

Moreover, previous studies [11-13] showed that left ventricular (LV) diastolic function diminished before systolic function and that this functional disorder is localized [11-13]. There are several studies using classical transmitral flow pulsed wave Doppler (PWD) in SCF patients, and these studies showed defective LV filling and diastolic function $[9,10]$.

Tissue Doppler echocardiography (TDE) is a technique derived by modifications from the PWD technique and is a popular method to quantitatively evaluate global or local systolic and diastolic functions of the ventricles. Due to the limitations of the PWD method in diastolic function disorders, the TDE technique is becoming more popular [14].

Myocardial performance index (MPI) is an important parameter that can be calculated using classical PWD and TDE which reflects both diastolic and systolic functions. Elevated MPI values reflect poor prognosis in some patient groups [15-17]. In the literature, there are many studies $[9,18]$ evaluating LV function in SCF patients by PWD, but studies using TDE to evaluate LV function in SCF patients are rare [19]. In addition, there is only 1 study in the published literature about evaluating right ventricular (RV) function in SCF patients by TDE or MPI methods. In this study, we aimed to evaluate both ventricular functions using TDE and MPI methods in patients with SCF and to determine the relationship between these parameters and TFC in SCF patients.

\section{Subjects and Methods}

\section{Study Population}

Thirty-five patients (20 males and 15 females) with SCF who underwent coronary angiography after having typical angina and/or who had been diagnosed with SCF and 35 age- and sex-matched controls (14 males and 21 females) without SCF who underwent elective coronary angiography were enrolled in the study. Approval was obtained from the Institution Ethics Committee for this study. Written informed consent was obtained from all the enrolled patients. Exclusion criteria were patients with moderate to severe valvular heart disease, prosthetic heart valve, bundle branch block, atrial fibrillation, paced rhythm, atrioventricular block, restrictive, hypertrophic or dilated cardiomyopathies, congenital heart disease, coronary artery ectasia, major coronary spasm, previous history of myocardial infarction, uncontrolled hypertension, hyperthyroidism, hypothyroidism, malignancy, pulmonary, hepatic, renal and hematological disorders, and poor echocardiographic image. 

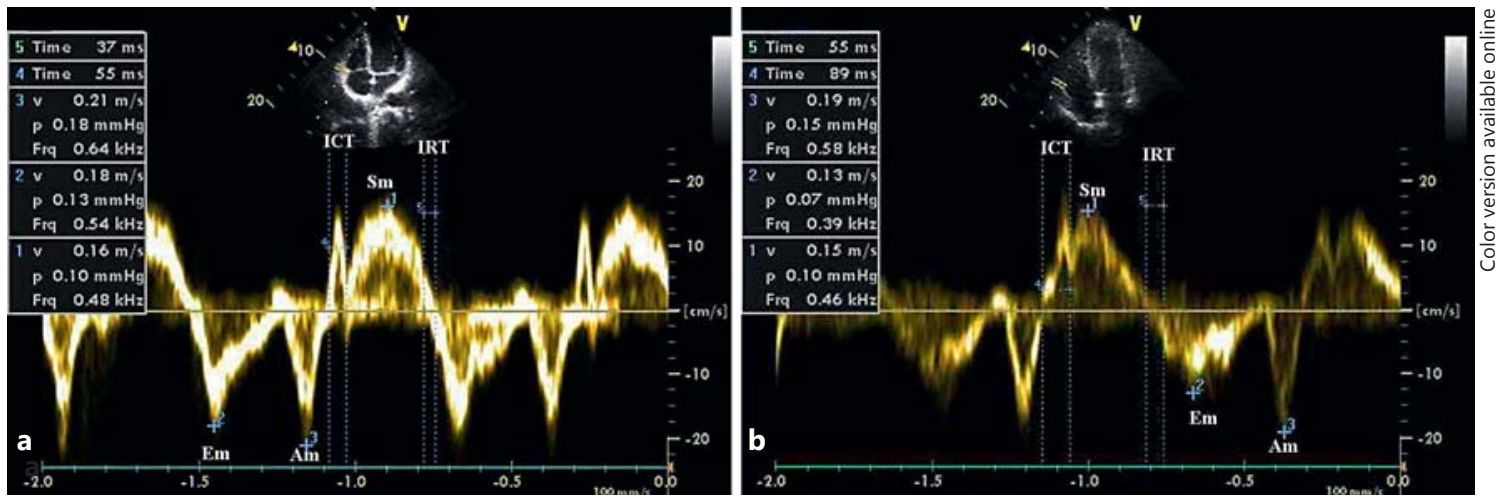

Fig. 3. RV tissue Doppler velocities. Assessment of RV Doppler velocities in the control (a) and SCF (b) groups. ICT $=$ Isovolumetric contraction time.

\section{Coronary Angiography and Analysis of TFC}

Coronary angiography was performed on all patients using the General Electric (GE) Innova 3100 (Milwaukee, Wisc., USA) with the standard Judkins technique in multiple angulated views. During coronary angiography, iohexol $350 / 100 \mathrm{ml}$ was used as a contrast agent and manually injected (5-6 $\mathrm{ml}$ at each position) in all patients. The diagnosis of SCF was made using the TFC method [8]. The number of cineangiographic frames, recording 30 frames per second, required for the leading edge of the column of radiographic contrast to first reach standard distal coronary landmarks in the left anterior descending (LAD), circumflex (CX), and right coronary arteries (RCAs) was recorded using the cine viewer frame counter. Subtracting the first frame number from the last frame number, TFC was evaluated for each artery. Because the corrected LAD (cLAD) coronary artery is typically longer than the CX and RCA, and the TFC for the LAD artery is often higher, the LAD artery frame counts were corrected by dividing by 1.7 to derive the corrected TFC as described earlier [8]. The TFCs in the $\mathrm{LAD}$ and $\mathrm{CX}$ arteries were assessed in a right anterior oblique projection with caudal angulation and the RCAs in left anterior oblique projection with cranial angulation (fig. 1,2). Two separate cardiologists (F.K. and K.C.) independently measured TFC in each patient.

\section{Echocardiography}

PWD and TDE were performed for all patients using a 2.5$\mathrm{MHz}$ transducer (Philips, EnVisor C Ultrasound, Bothell, Wash., USA) in the left decubitus position during normal respiration according to the recommendations of the American Society of Echocardiography [20]. LV diameter and thicknesses were measured from the parasternal window with two-dimensional M-mode echocardiography. LV ejection fraction was measured by the modified biplane Simpson's method [21]. RV diameters were measured from the apical 4-chamber view. Doppler recordings were obtained with the pulsed sample volume placed at the tip of the mitral leaflets from the apical 4-chamber view. Peak early and late velocities, E-wave deceleration time and isovolumetric relaxation time (IRT) were measured. Pulmonary venous flow was measured from the apical 4-chamber view by placing the PWD sample volume $1 \mathrm{~cm}$ into the right upper pulmonary vein. The peak pulmonary vein systolic velocity (Ps) and the peak pulmonary vein diastolic velocity $(\mathrm{Pd})$ were measured, and the Ps/Pd ratio was calculated. TDE parameters were measured using an echocardiographic device with active TDE functions (Philips, EnVisor C Ultrasound). The filter and gain settings were adjusted to the minimal optimal level to reduce noise and eliminate the signal produced by flow. A $3.5-\mathrm{mm}$ sample volume was used. From the apical 4-chamber view, the Doppler sample volume was placed at the lateral corner of the mitral annulus and lateral corner of the tricuspid annulus. A Doppler velocity range of -20 to $20 \mathrm{~cm} / \mathrm{s}$ was selected, and the velocities were measured online at a sweep of $100 \mathrm{~mm} / \mathrm{s}$. Peak systolic myocardial velocity (Sm), peak early myocardial velocity (Em), and late myocardial velocity (Am) were measured for the lateral segment and the Em/Am ratio was calculated. The IRT was measured from the end of Sm to the beginning of Em, the isovolumetric contraction time was measured from the end of Am to the beginning of Sm, and the time period of Sm was measured as the ejection time (fig. 3). The MPI was calculated using the equation (isovolumetric contraction time + IRT)/ejection time [22]. The Sm, Em, and Am values obtained from the tricuspid annulus were used for the RV MPI. All Doppler parameters were obtained by calculating the mean of 5 consecutive cycles. Echocardiography measurements were made by the same cardiologist (F.A.), who was blinded to the clinical and angiographic features of the patients.

\section{Statistical Analysis}

Continuous variables are expressed as means \pm standard deviation (SD) and categorical variables are given as percentages. The Shapiro-Wilk test was used to evaluate whether the distribution of variables was normal. A two-sided independent samples t test or the Mann-Whitney U test was used to compare continuous variables between the two groups. The $\chi^{2}$ test, $\chi^{2}$ with continuity correction, or Fisher's exact test was applied for the categorical variables when necessary. Pearson's $r$ correlation coefficients were used to evaluate correlation between variables. SPSS software 11.0 for Windows (Chicago, Ill., USA) was used for all statistical analyses. Calculated $\mathrm{p}$ values were considered statistically significant at $\mathrm{p}<0.05$.
Altunkas et al. 


\section{Results}

The baseline characteristics and angiographic findings of the SCF and control groups are given in table 1. There were no significant differences in baseline characteristics among the groups; however, TFC values were higher in the SCF group compared to the control group.

The two-dimensional echocardiography and PWD parameters of the left ventricle and the two-dimensional echocardiography parameters of the right ventricle are listed in table 2 for the SCF and control groups. There were no significant differences in early diastolic mitral inflow (E) velocity, late diastolic mitral inflow (A) velocity, E/A ratio, deceleration time of the mitral valve, left atrial, $\mathrm{LV}$ and RV dimensions, ejection fraction, and LV mass among the groups.

The TDE parameters obtained from the left and right ventricles are given in table 3. LV Sm, Em, Am, and Em/ Am were similar in both the SCF and control groups; however, IRT and MPI were higher in the SCF group compared to the control group (IRT: $99 \pm 17$ vs. $88 \pm 20$; $=$ 0.01 , and MPI: $0.61 \pm 0.11$ vs. $0.56 \pm 0.12 ; \mathrm{p}=0.07)$. RV wall Sm, Em, Am, IRT, and MPI were similar in both the SCF and control groups. RCA TFC and cLAD artery TFC were positively correlated with LV lateral wall IRT $(\mathrm{r}=$ $0.243, p=0.04$ and $r=0.233, p<0.05$, respectively). In addition, CLAD artery TFC, LAD artery TFC, and RCA TFC were positively correlated with LV MPI $(\mathrm{r}=0.212, \mathrm{p}=$ $0.08 ; \mathrm{r}=0.212, \mathrm{p}=0.08$, and $\mathrm{r}=0.214, \mathrm{p}=0.07$, respectively), but this correlation was not statistically significant.

\section{Discussion}

In this study, RV MPI was similar in the two groups. The LV IRT was significantly higher in the SCF group than in the control group. LV MPI was higher in the SCF group than in the control group, but this difference was not statistically significant. LV diastolic function disorder is the cardiac pathology with the earliest onset in SCF. In our study, no statistically significant difference between LV diastolic functions of the control group and the SCF group was detected by conventional Doppler echocardiography. However, Bonow et al. [23] reported that the earliest indicator of myocardial ischemia is LV relaxation disorder, a parameter of diastolic function. Sezgin et al. [9] and Tanriverdi et al. [18] also showed that there is LV relaxation disorder in SCF patients using conventional Doppler echocardiography. Despite these studies, Nurkalem et al. [24] and Baykan et al. [19] found no LV relaxation disorder in their study on SCF patients using the same methods.

Slow Coronary Flow and Ventricular

Performance
Table 1. Demographic and angiographic characteristics in the SCF and control groups

\begin{tabular}{|c|c|c|c|}
\hline & $\begin{array}{l}\text { SCF } \\
(n=35)\end{array}$ & $\begin{array}{l}\text { Controls } \\
(\mathrm{n}=35)\end{array}$ & $\mathrm{p}$ \\
\hline Age, years & $55 \pm 11$ & $54 \pm 9$ & 0.79 \\
\hline Male sex & $20(57)$ & $14(40)$ & 0.15 \\
\hline Body mass index & $31 \pm 4$ & $30 \pm 4$ & 0.17 \\
\hline Systolic blood pressure, $\mathrm{mm} \mathrm{Hg}$ & $126 \pm 19$ & $124 \pm 22$ & 0.65 \\
\hline Diastolic blood pressure, $\mathrm{mm} \mathrm{Hg}$ & $80 \pm 12$ & $77 \pm 12$ & 0.29 \\
\hline Hypertension & $19(54)$ & $14(40)$ & 0.23 \\
\hline Hyperlipidemia & $18(51)$ & $12(34)$ & 0.15 \\
\hline Diabetes mellitus & $5(14)$ & $9(26)$ & 0.23 \\
\hline Smoking & $6(17)$ & $7(20)$ & 0.76 \\
\hline Family history & $7(20)$ & $4(11)$ & 0.32 \\
\hline \multicolumn{4}{|l|}{ Medications } \\
\hline ACEI/ARB & $14(40)$ & $10(29)$ & 0.31 \\
\hline$\beta$-Blockers & $10(29)$ & $9(26)$ & 0.79 \\
\hline Nitrates & $6(17)$ & $3(9)$ & 0.48 \\
\hline Calcium antagonists & $2(6)$ & $4(11)$ & 0.67 \\
\hline Statin & $11(31)$ & $6(17)$ & 0.16 \\
\hline Fasting serum glucose, $\mathrm{mg} / \mathrm{dl}$ & $102 \pm 23$ & $105 \pm 17$ & 0.3 \\
\hline Creatinin, mg/dl & $0.74 \pm 0.18$ & $0.72 \pm 0.17$ & 0.59 \\
\hline Total cholesterol, mg/dl & $204 \pm 43$ & $202 \pm 43$ & 0.824 \\
\hline Triglycerides, mg/dl & $176 \pm 141$ & $168 \pm 82$ & 0.49 \\
\hline HDL cholesterol, mg/dl & $43 \pm 12$ & $43 \pm 11$ & 0.803 \\
\hline LDL cholesterol, mg/dl & $124 \pm 31$ & $129 \pm 34$ & 0.53 \\
\hline \multicolumn{4}{|l|}{ Vessels with slow flow } \\
\hline One vessel & $5(14)$ & $0(0)$ & 0.05 \\
\hline Two vessels & $14(40)$ & $0(0)$ & $<0.001$ \\
\hline Three vessels & $16(46)$ & $0(0)$ & $<0.001$ \\
\hline \multicolumn{4}{|l|}{ TFC } \\
\hline LAD & $59 \pm 19$ & $34 \pm 3$ & $<0.001$ \\
\hline $\mathrm{Cx}$ & $30 \pm 10$ & $22 \pm 2$ & $<0.001$ \\
\hline RCA & $30 \pm 9$ & $20 \pm 2$ & $<0.001$ \\
\hline cLAD & $35 \pm 11$ & $20 \pm 2$ & $<0.001$ \\
\hline Mean & $32 \pm 10$ & $21 \pm 2$ & $<0.001$ \\
\hline
\end{tabular}

Values are means \pm SD or numbers with percentages in parentheses. $\mathrm{ACEI}=$ Angiotensin-converting enzyme inhibitor; $\mathrm{ARB}=$ angiotensin II receptor blocker; LDL = low-density lipoprotein; $\mathrm{HDL}=$ high-density lipoprotein.

Myocardial velocities obtained using TDE are considered to be new parameters to evaluate LV functions [25]. A study has shown that TDE parameters are not affected by preload and heart rate, like conventional Doppler, and thus yield more accurate results [26]. In our study, the differences in LV Sm, Em, and Am velocities and $\mathrm{Em} / \mathrm{Am}$ ratio were not statistically significant between the control and SCF groups, and only the LV IRT value was significantly longer in the SCF group compared to the control group. A probable explanation 
Table 2. Two-dimensional M-mode and PWD echocardiography in the SCF and control groups

\begin{tabular}{lccl}
\hline & SCF $(\mathrm{n}=35)$ & Controls $(\mathrm{n}=35)$ & $\mathrm{p}$ \\
\hline LVEDD, cm & $5 \pm 0.6$ & $4.8 \pm 0.4$ & 0.17 \\
LVESD, cm & $3.3 \pm 0.8$ & $3 \pm 0.4$ & 0.12 \\
IVS, cm & $1.1 \pm 0.2$ & $1 \pm 0.2$ & 0.86 \\
EF, \% & $59.6 \pm 1.4$ & $59.7 \pm 1.4$ & 0.87 \\
PW, cm & $1 \pm 0.2$ & $0.9 \pm 0.1$ & 0.23 \\
RVEDD, cm & $3.2 \pm 0.6$ & $3.2 \pm 0.4$ & 0.91 \\
RVESD, cm & $2.2 \pm 0.6$ & $2.1 \pm 0.3$ & 0.15 \\
LA, cm & $3.6 \pm 0.7$ & $3.5 \pm 0.4$ & 0.43 \\
LV mass, g & $223 \pm 63$ & $202 \pm 55$ & 0.28 \\
E, cm/s & $65 \pm 16$ & $69 \pm 20$ & 0.41 \\
A, cm/s & $73 \pm 19$ & $80 \pm 26$ & 0.3 \\
DT, ms & $200 \pm 43$ & $201 \pm 42$ & 0.95 \\
E/A & $0.95 \pm 0.33$ & $0.91 \pm 0.32$ & 0.58 \\
E/Em & $9.5 \pm 2.4$ & $10.1 \pm 4.2$ & 0.45 \\
Ps/Pd & $1.29 \pm 0.3$ & $1.27 \pm 0.31$ & 0.75 \\
Ra, cm/s & $25 \pm 4$ & $26 \pm 6$ & 0.7 \\
\hline
\end{tabular}

Values are means \pm SD. LVEDD $=$ Left ventricular end-diastolic diameter; LVESD = left ventricular end-systolic diameter; IVS = interventricular septum; $\mathrm{EF}=$ ejection fraction; $\mathrm{PW}=$ posterior wall; RVEDD = right ventricular end-diastolic diameter; RVESD = right ventricular end-systolic diameter; LA = left atrial diameter; DT $=$ deceleration time of the mitral valve; $\mathrm{Ra}=$ pulmonary venous atrial reversal wave.

Table 3. Tissue Doppler imaging measurements in the SCF and control groups

\begin{tabular}{lccc}
\hline & SCF $(\mathrm{n}=35)$ & Controls $(\mathrm{n}=35)$ & $\mathrm{p}$ \\
\hline Left ventricle & & & \\
Sm, cm/s & $8.2 \pm 1.3$ & $7.9 \pm 1.3$ & 0.39 \\
Em, cm/s & $7.1 \pm 1.9$ & $7.3 \pm 2$ & 0.73 \\
Am, cm/s & $11 \pm 2.2$ & $10.2 \pm 1.7$ & 0.13 \\
Em/Am & $0.69 \pm 0.31$ & $0.73 \pm 0.23$ & 0.53 \\
IRT, ms & $99 \pm 17$ & $88 \pm 20$ & 0.01 \\
MPI & $0.61 \pm 0.11$ & $0.56 \pm 0.12$ & 0.07 \\
Right ventricle & & & \\
Sm, cm/s & $13.5 \pm 2.6$ & $12.4 \pm 2.5$ & 0.08 \\
Em, cm/s & $9.8 \pm 3.1$ & $9.3 \pm 2.6$ & 0.54 \\
Am, cm/s & $13.9 \pm 3$ & $17.2 \pm 19$ & 0.42 \\
IRT, ms & $83 \pm 28$ & $74 \pm 29$ & 0.21 \\
MPI & $0.52 \pm 0.17$ & $0.51 \pm 0.18$ & 0.93 \\
\hline
\end{tabular}

Values are means \pm SD. could be due to the markedly higher mean TCF values of the patients in the Baykan et al. [19] study (mean TFC: $41 \pm 8$ vs. $32 \pm 10$, respectively). In their study on SCF patients using the TDE method, Sevimli et al. [10] showed that LV relaxation disorder is evident. In this study, TFC, PWD, and TDE are used to determine diastolic function parameters and show significant correlation. Baykan et al. [19] used both conventional and TDE methods to evaluate the systolic and diastolic functions of SCF patients. The conventional method showed no significant difference between systolic and diastolic functions of the two groups; however, using the TDE method for evaluation of LV functions revealed that $\mathrm{Sm}$ velocity, Em velocity, and Em/Am ratio of SCF patients was significantly lower than in the control group. In their study, Yllmaz et al. [27] used conventional and TDE methods to evaluate the RV function in patients with SCF. In this study, RV Em and Em/Am ratio were lower than in the control group and RV Am and RV IRT were higher than in the control group.

MPI, calculated by conventional echocardiography and TDE methods, is a useful parameter to evaluate systolic and diastolic functions of the ventricles. In our study, we observed marked increased LV MPI values in SCF patients. We also observed a positive correlation between LV MPI and CLAD and RCA, but this correlation was not statistically significant. In the literature, there are few studies evaluating the relationship between LV functions, coronary artery TFC numbers, and MPI in SCF patients. In their study, Baykan et al. [19] assessed this relationship and reported a statistically significant increase in the MPI values of SCF patients compared to a control group. Also in this study, a positive and statistically significant correlation between mean TFC, RCA TFC, and MPI was established [19]. We have concluded that the reason our results failed to reach statistical significance may be low mean TFC values relative to the Baykan et al. [19] study (32 \pm 10 vs. $41 \pm 8$, respectively). Both our study and the Baykan et al. [19] study showed a correlation between MPI, diastolic functional parameters, and TFC.

RV functions are considered less important than LV functions and have become of secondary importance in clinical practice. Even though it is called the 'forgotten ventricle' in some publications, the right ventricle has great importance in the prognosis of patients with LV dysfunction and congenital heart diseases [28]. Gondi et al. [28] define the RV Sm value as an independent indicator of clinical endpoints in patients with LV heart failure. There is only 1 study in the published literature evaluating RV performance in SCF patients by the TDE or MPI method. 
Our study is important since it is the second study on this subject. However, we observed no statistically significant difference between diastolic functions and RV MPI values. In the first study, Yllmaz et al. [27] observed significantly increased RV MPI values in SCF patients.

\section{Study Limitations}

First, relatively few patients were included in this study. In the future, the number of participating centers should be increased and the results should be confirmed by more comprehensive studies. Second, obtaining LV TDE measurements only from the lateral wall may be a limitation. Increasing the number of wall measurements to an average of 2-4 in this study may have been more ideal. Third, the TFC values of the patients in our study were higher than in the control group but lower than the values in the literature. This decreases the statistical value of our study.

\section{Conclusion}

When considered together with the results of other studies, our results provide further evidence that in patients with normal coronary arteries, there is a statistically significant correlation between SCF and impaired LV function. Our results failed to demonstrate a similar relationship between SCF and RV function.

\section{References}

$>1$ Tambe AA, Demany MA, Zimmerman HA, et al: Angina pectoris and slow flow velocity of dye in coronary arteries - a new angiographic finding. Am Heart J 1972;84:66-71.

$>2$ Sezgin AT, Sigirci A, Barutcu I, et al: Vascular endothelial function in patients with slow coronary flow. Coron Artery Dis 2003;14: 155-161.

3 Kalay N, Aytekin M, Kaya MG, et al: The relationship between inflammation and slow coronary flow: increased red cell distribution width and serum uric acid levels. Turk Kardiyol Dern Ars 2011;39:463-468.

4 Mangieri E, Macchiarelli G, Ciavolella M, et al: Slow coronary flow: clinical and histopathological features in patients with otherwise normal epicardial coronary arteries. Cathet Cardiovasc Diagn 1996;37:375-381.

5 Luscinskas FW, Gimbrone MA Jr: Endothelial-dependent mechanisms in chronic inflammatory leukocyte recruitment. Annu Rev Med 1996;47:413-421.

6 Vogel RA: Measurement of endothelial function by brachial artery flow-mediated vasodilation. Am J Cardiol 2001;88:31E-34E.

7 Goel PK, Gupta SK, Agarwal A, et al: Slow coronary flow: a distinct angiographic subgroup in syndrome X. Angiology 2001;52: 507-514.

8 Gibson CM, Cannon CP, Daley WL, et al: TIMI frame count: a quantitative method of assessing coronary artery flow. Circulation 1996;93:879-888.

$>9$ Sezgin AT, Topal E, Barutcu I, et al: Impaired left ventricle filling in slow coronary flow phenomenon: an echo-Doppler study. Angiology 2005;56:397-401.

10 Sevimli S, Büyükkaya E, Gündoğdu F, et al: Left ventricular function in patients with coronary slow flow: a tissue Doppler study. Arch Turk Soc Cardiol 2007;35:360-365.

Slow Coronary Flow and Ventricular Performance
11 Mandinov L, Eberli FR, Seiler C, et al: Diastolic heart failure. Cardiovasc Res 2000;45: 813-825.

12 Garcia-Fernandez MA, Azevedo J, Moreno M, et al: Regional diastolic function in ischaemic heart disease using pulsed wave Doppler tissue imaging. Eur Heart J 1999;20:496-505.

13 Bach DS, Armstrong WF, Donovan CL, et al: Quantitative Doppler tissue imaging for assessment of regional myocardial velocities during transient ischemia and reperfusion. Am Heart J 1996;132:721-725.

14 Nishimura RA, Housmans PR, Hatle LK, et al: Assessment of diastolic function of the heart: background and current applications of Doppler echocardiography. Part I. Physiologic and pathophysiologic features. Mayo Clin Proc 1989;64:71-81.

15 Dujardin KS, Tei C, Yeo TC, et al: Prognostic value of a Doppler index combining systolic and diastolic performance in idiopathic-dilated cardiomyopathy. Am J Cardiol 1998;82: 1071-1076.

16 Yeo TC, Dujardin KS, Tei C, et al: Value of a Doppler-derived index combining systolic and diastolic time intervals in predicting outcome in primary pulmonary hypertension. Am J Cardiol 1998;81:1157-1161.

17 Ceyhan K, Koc F, Ozdemir K, et al: Coronary ectasia is associated with impaired left ventricular myocardial performance in patients without significant coronary artery stenosis. Med Princ Pract 2012;21:139-144.

18 Tanriverdi H, Evrengul H, Kilic ID, et al: Aortic pressures, stiffness and left ventricular function in coronary slow flow phenomenon. Cardiology 2010;116:261-267.

19 Baykan M, Baykan EC, Turan S, et al: Assessment of left ventricular function and Tei in dex by tissue Doppler imaging in patients with slow coronary flow. Echocardiography 2009;26:1167-1172.
20 Nagueh SF, Appleton CP, Gillebert TC, et al: Recommendations for the evaluation of left ventricular diastolic function by echocardiography. Eur J Echocardiogr 2009;10:165-193.

21 Lang RM, Bierig M, Devereux RB, et al: Recommendations for chamber quantification: a report from the American Society of Echocardiography's Guidelines and Standards Committee and the Chamber Quantification Writing Group. J Am Soc Echocardiogr 2005;18: 1440-1463.

22 Tei C, Nishimura RA, Seward JB, et al: Noninvasive Doppler-derived myocardial performance index: correlation with simultaneous measurements of cardiac catheterization measurements. J Am Soc Echocardiogr 1997; 10:169-178.

-23 Bonow RO, Kent KM, Rosing DR, et al: Improved left ventricular diastolic filling in patients with coronary artery disease after percutaneous transluminal coronary angioplasty. Circulation 1982;66:1159-1167.

24 Nurkalem Z, Gorgulu S, Uslu N, et al: Longitudinal left ventricular systolic function is impaired in patients with coronary slow flow. Int J Cardiovasc Imaging 2009;25:25-32.

25 Ozdemir K, Altunkeser BB, Içli A, et al: New parameters in identification of right ventricular myocardial infarction and proximal right coronary artery lesion. Chest 2003;124:219-226.

26 Ozdemir K, Balci S, Duzenli MA, et al: Effect of preload and heart rate on the Doppler and tissue Doppler-derived myocardial performance index. Clin Cardiol 2007;30:342-348.

27 Yilmaz M, Ozluk F, Peker T, et al: Right ventricular function and its relation with TIMI frame count in the coronary slow flow phenomenon. Turk J Med Sci 2013;43:46-51.

28 Gondi S, Dokainish H: Right ventricular tissue Doppler and strain imaging: ready for clinical use? Echocardiography 2007;24:522532

Performance 